\title{
Alcohol Inhibits Odontogenic Differentiation of Human Dental Pulp Cells by Activating mTOR Signaling
}

\author{
Wei Qin, ${ }^{1,2}$ Qi-Ting Huang, ${ }^{1,2}$ Michael D. Weir, ${ }^{2}$ Zhi Song, ${ }^{1}$ Ashraf F. Fouad, ${ }^{3}$ \\ Zheng-Mei Lin, ${ }^{1}$ Liang $\mathrm{Zhao},{ }^{2,4}$ and Hockin H. K. $\mathrm{Xu}^{2,5,6}$ \\ ${ }^{1}$ Department of Operative Dentistry and Endodontics, Guanghua School of Stomatology, Sun Yat-sen University and Guangdong \\ Provincial Key Laboratory of Stomatology, Guangzhou 510055, China \\ ${ }^{2}$ Biomaterials and Tissue Engineering Division, Department of Endodontics, Periodontics and Prosthodontics, University of \\ Maryland School of Dentistry, Baltimore, MD 21201, USA \\ ${ }^{3}$ Department of Endodontics, School of Dentistry, University of North Carolina, Chapel Hill, NC 27599-7450, USA \\ ${ }^{4}$ Department of Orthopedic Surgery, Nanfang Hospital, Southern Medical University, Guangzhou, Guangdong 510515, China \\ ${ }^{5}$ Center for Stem Cell Biology \& Regenerative Medicine, University of Maryland School of Medicine, Baltimore, MD 21201, USA \\ ${ }^{6}$ Department of Mechanical Engineering, University of Maryland, Baltimore County, Baltimore County, MD 21250, USA
}

Correspondence should be addressed to Zheng-Mei Lin; linzhm@mail.sysu.edu.cn, Liang Zhao; lzhaonf@126.com, and Hockin H. K. Xu; hxu@umaryland.edu

Received 20 April 2017; Revised 5 July 2017; Accepted 16 July 2017; Published 14 September 2017

Academic Editor: Antonio Boccaccio

Copyright ( 2017 Wei Qin et al. This is an open access article distributed under the Creative Commons Attribution License, which permits unrestricted use, distribution, and reproduction in any medium, provided the original work is properly cited.

Long-term heavy alcohol consumption could result in a range of health, social, and behavioral problems. People who abuse alcohol are at high risks of seriously having osteopenia, periodontal disease, and compromised oral health. However, the role of ethanol $(\mathrm{EtOH})$ in the biological functions of human dental pulp cells (DPCs) is unknown. Whether EtOH affects the odontoblastic differentiation of DPCs through the mechanistic target of rapamycin (mTOR) remains unexplored. The objective of this study was to investigate the effects of EtOH on DPC differentiation and mineralization. DPCs were isolated and purified from human dental pulps. The proliferation and odontoblastic differentiation of DPCs treated with EtOH were subsequently investigated. Different doses of EtOH were shown to be cytocompatible with DPCs. EtOH significantly activated the mTOR pathway in a dose-dependent manner. In addition, EtOH downregulated the alkaline phosphatase activity, attenuated the mineralized nodule formation, and suppressed the expression of odontoblastic markers including ALP, DSPP, DMP-1, Runx2, and OCN. Moreover, the pretreatment with rapamycin, a specific mTOR inhibitor, markedly reversed the EtOH-induced odontoblastic differentiation and cell mineralization. Our findings show for the first time that EtOH can suppress DPC differentiation and mineralization in a mTOR-dependent manner, indicating that $\mathrm{EtOH}$ may be involved in negatively regulating the dental pulp repair.

\section{Introduction}

Alcohol is widely consumed throughout the world and has attracted human concernment for thousands of years. Alcohol abuse can place the health of an individual at risk for a series of diseases. According to the World Health Organization, heavy alcohol consumptions are associated with many chronic diseases, including low bone mass, hepatitis, and cardiovascular diseases [1]. Chronic and heavy alcohol consumption is known to result in bone loss, decreased bone formation, increased risks for bone fracture, and delayed fracture healing [2-4]. Moderate alcohol consumption may actually have a modest favorable effect on bone density, particularly in postmenopausal women, although not all studies agree [5-8]. However, alcohol intake of three or more drinks per day is detrimental to bone health [9]. Recent experimental evidences indicated that the mammalian target of rapamycin (mTOR) signal may contribute to the maintenance of bone homeostasis and the differentiation of mesenchymal stem cells $[10,11]$.

DPCs possess multipotent differentiation potential and the ability to form dentin-pulp-like complexes throughout 
life. When the dental pulp is confronted with trauma, microbes, or chemicals, a host of inflammatory cytokines are released [12]. These insults can stimulate the underlying progenitor pulp cells to differentiate into odontoblasts [13], which are capable of secreting dentin matrix proteins as part of the reparative dentinogenesis [14]. Odontoblasts secrete several collagenous and noncollagenous proteins, such as type I collagen, osteopontin, dentin matrix protein 1 (DMP1), and dentin sialophosphoprotein (DSPP), which are special biological markers for the odontoblast/osteoblast-like differentiation of DPCs $[15,16]$. Studies have shown that a variety of signal pathways participate in the regulation of dental pulp cell differentiation, such as BMP, Wnt, and Notch signaling [17-19]. However, the impact of alcohol on odontoblastic differentiation of human dental pulp cells (DPCs) remains unclear.

Therefore, the objective of the present study was to investigate the effects of ethanol $(\mathrm{EtOH})$ on the proliferation and odontoblastic differentiation of DPCs. The role of mTOR signaling in EtOH-mediated odontoblastic differentiation was also investigated. The results of this study will shed light on the role of alcohol consumption on the health of human dental pulp cells and their ability in tissue repair and regeneration.

\section{Materials and Methods}

2.1. Cell Cultures. DPCs were isolated and characterized as described previously $[20,21]$. Dental pulp tissues were obtained from explants of clinically healthy dental pulps from human adult third molars that were removed from individuals undergoing tooth extraction for orthodontic treatment. The procedure was approved by the Institutional Review Board of the University of Maryland Baltimore. The pulp tissue was digested in a solution of $3 \mathrm{mg} / \mathrm{mL}$ collagenase type I (Worthington Biochem, Freehold, NJ, USA) and $4 \mathrm{mg} / \mathrm{mL}$ dispase (Boehringer Mannheim, Indianapolis, IN, USA) for $1 \mathrm{~h}$ at $37^{\circ} \mathrm{C}$. In the present study, DPCs were cultured in alpha modified Eagle's medium (Invitrogen, Carlsbad, CA, USA) supplemented with $10 \%$ foetal calf serum (FCS; Invitrogen), $10 \mathrm{mM}$ L-ascorbic acid 2phosphate (AA), $2 \mathrm{mM}$ L-glutamate, 100 units/mL penicillin, and $100 \mu \mathrm{g} / \mathrm{mL}$ streptomycin at $37^{\circ} \mathrm{C}$ in a humidified atmosphere of $5 \% \mathrm{CO}_{2}$ and $95 \%$ air. When the cells reached $80 \%$ confluence, they were harvested using trypsin/ethylene diamine tetraacetic acid (EDTA) (Gibco, Carlsbad, CA, USA) and subcultured at a ratio of $1: 3$. The 4 th passage DPCs were used in the following experiments.

2.2. Cell Viability. DPCs were seeded in 24-well plates at a density of $3 \times 10^{4}$ cells/well. Cells were stained by live/dead viability assay kit (Life Technologies) after culture for 1, 7, and $14 \mathrm{~d}$ as described previously [22]. Cells were washed with PBS, followed by incubation with the dye. Live cells were stained green with $2 \mathrm{mM}$ calcein AM and dead cells were marked red with $4 \mathrm{mM}$ ethidium homodimer-1, and they were examined using epifluorescence microscopy (Eclipse TE2000-S, Nikon, Melville, NY). The percentage of live cells and the live cell density were calculated as previously described [21]. Three random sections were analyzed for each sample.

2.3. Cell Proliferation Assays. A cell counting kit (CCK-8, Dojindo, Tokyo, Japan) was used to evaluate cell proliferation at 1, 3, 5, and $7 \mathrm{~d}$. Four replicates in each group were used for this assay. CCK- 8 is based on the WST- 8 reaction that produces an orange formazan dye in an amount that is directly related with the number of viable cells. The cell proliferative rate was determined via the absorbance at an optical density of $450 \mathrm{~nm}\left(\mathrm{OD}_{450 \mathrm{~nm}}\right)$ using a microplate reader (SpectraMax M5, Molecular Devices, Sunnyvale, CA) according to the manufacturer's protocol.

2.4. Western Blot Analysis. Cells were harvested and lysed in lysis buffer: $20 \mathrm{mmol} / \mathrm{L} \mathrm{Na}_{2} \mathrm{PO}_{4}$ at $\mathrm{pH} 7.4,150 \mathrm{mmol} / \mathrm{L} \mathrm{NaCl}$, $1 \%$ Triton $\mathrm{X}-100,1 \%$ aprotinin, $1 \mathrm{mmol} / \mathrm{L}$ phenymethysulfonyl fluoride, $10 \mathrm{mg} / \mathrm{mL}$ leupeptin, $100 \mathrm{mmol} / \mathrm{L} \mathrm{NaF}$, and $2 \mathrm{mmol} / \mathrm{L} \mathrm{Na}_{3} \mathrm{VO}_{4}$. Lysates were centrifuged at 12,000 rpm for $15 \mathrm{~min}$. The supernatant was collected, and the protein content was determined using the Bio-Rad protein assay. SDS-PAGE sample buffer (10 mM Tris- $\mathrm{HCl}, \mathrm{pH} 6.8,2 \%$ SDS, $10 \%$ glycerol, and $0.2 \mathrm{M} \mathrm{DTT}$ ) was added to the lysates. Lysates were heated to $100^{\circ} \mathrm{C}$ for $8 \mathrm{~min}$, and $20 \mu \mathrm{g}$ of the total protein was loaded in each well of a 10\% SDS-PAGE gel. Western blot analysis was performed as reported previously $[23,24]$. The following primary antibodies were used: phospho-mTOR (p-mTOR), and total mTOR antibody (Cell Signaling Technology, Beverly, MA, USA).

2.5. Alkaline Phosphatase Activity. DPCs were preincubated with $50 \mathrm{mM}$ rapamycin 1 hour and then exposed to $50 \mathrm{mM}$ EtOH; this procedure was repeated at day 3. After the treatment, the cells were scraped into cold PBS and then sonicated in an ice bath and centrifuged at $1500 \times \mathrm{g}$ for $5 \mathrm{~min}$. Then, the ALP activity was measured in the supernatant using ALP assay mixtures containing $0.1 \mathrm{M}$ diethanolamine, $1 \mathrm{mM}$ $\mathrm{MgCl}_{2}$, and $10 \mathrm{mg} / \mathrm{mL}$ p-nitrophenyl phosphate. After incubation at $37^{\circ} \mathrm{C}$ for $30 \mathrm{~min}$, the reaction was stopped by the addition of $\mathrm{NaOH}$, and the absorbance was measured at $410 \mathrm{~nm}$ using the microplate reader (SpectraMax M5).

2.6. Reverse Transcriptase PCR (RT-PCR) and Real-Time Quantitative PCR ( $P P C R)$. The expression levels of ALP, DSPP, DMP-1, Runx2, and OCN mRNA were determined by SYBR green real-time reverse transcription-PCR (RTPCR) as described previously [25]. Total RNA were extracted using TRIzol reagent. Quantitative determination of RNA levels were performed in triplicate in three independent experiments. Real-time PCR and data collection were performed with an ABI PRISM 7500 sequence detection system. The housekeeping gene GAPDH was used as an internal control to normalize the expression levels of different genes. For each primer set, the melting curves were performed to ensure that a single peak was produced. The data for gene expression were analyzed with the $\triangle \triangle \mathrm{Ct}$ method. The primers used for the amplification of the indicated genes are listed in Table 1.

2.7. Von Kossa Staining. Specific calcifications were detected by von Kossa staining $[26,27]$. Briefly, DPCs were plated in 
TABLE 1: List of reverse transcriptase polymerase chain reaction primers.

\begin{tabular}{lrc}
\hline Gene & Forward & Reverse \\
\hline ALP & CTATCCTGGCTCCGTGCTC & GCTGGCAGTGGTCAGATGTT \\
DSPP & TGGAGCCACAAACAGAAGCAA & TCCAGCTACTTGAGGTCCATC \\
DMP-1 & GTGAGTGAGTCCAGGGGAGATAA & TTTTGAGTGGGAGAGTGTGTGC \\
Runx2 & GACTGTGGTTACCGTCATGGC & ACTTGGTTTTTCATAACAGCGGA \\
OCN & CTCACACTCCTCGCCCTATT & TTGGACACAAAGGCTGCAC \\
GAPDH & TCAACGACCCCTTCATTGAC & ATGCAGGGATGATGTTCTGG \\
\hline
\end{tabular}
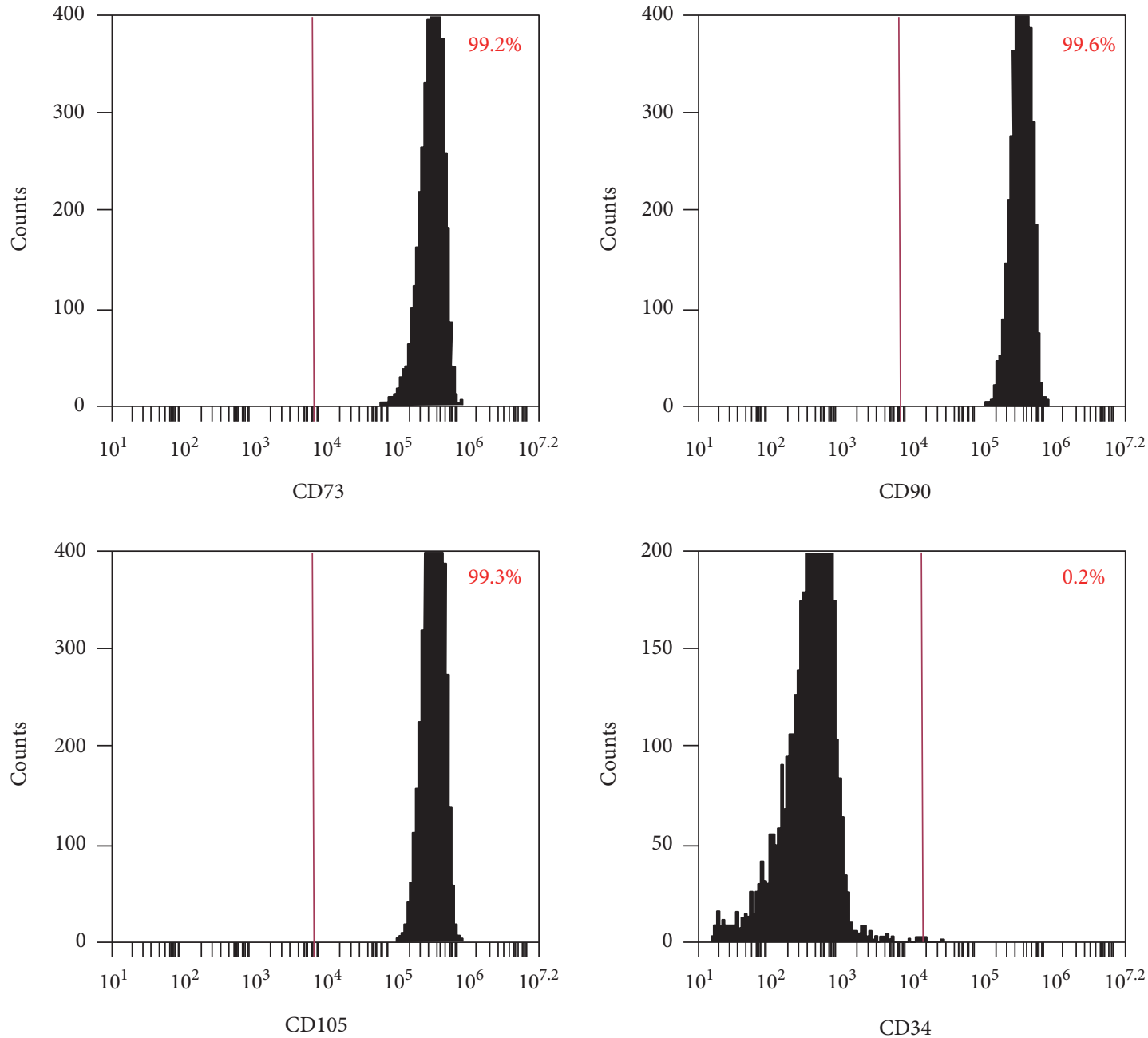

FIGURE 1: DPCs phenotype via flow cytometry. The expression of a series of cell surface markers associated with the mesenchymal stem cell (MSC) phenotype was investigated using flow cytometry. Analysis of molecular surface antigen markers in DPCs by flow cytometry indicated that the cells were negative for CD34, whereas they were positive for CD73, CD90, and CD105.

six-well plates at a density of $1 \times 10^{5}$ cells per well and cultured in DMEM supplemented with $10 \% \mathrm{FBS}, 50 \mathrm{mg} / \mathrm{mL}$ ascorbic acid, $10 \mathrm{mmol} / \mathrm{L}$ sodium $\beta$-glycerophosphate, and $10 \mathrm{nmol} / \mathrm{L}$ dexamethasone. DPCs were then pretreated with $50 \mathrm{mM}$ rapamycin for 1 hour prior to the addition of $50 \mathrm{mM}$ EtOH. This treatment was repeated every $3 \mathrm{~d}$. After $14 \mathrm{~d}$ of treatment, the cells were treated with a $5 \%$ silver nitrate solution and exposed to ultraviolet light for $30 \mathrm{~min}$.
This solution was then neutralized with $5 \%$ sodium thiosulfate for $2 \mathrm{~min}$, and the cells were rinsed with distilled water for $5 \mathrm{~min}$. Finally, the cells were stained with nuclear fast red (Sigma, Deisenhofen, Germany) for $1 \mathrm{~min}$. To quantify the mineralization, the calcium content was measured by a quantitative colorimetric method using a calcium assay kit following the manufacturer's instructions. The calcium content of the cell layer was determined at 

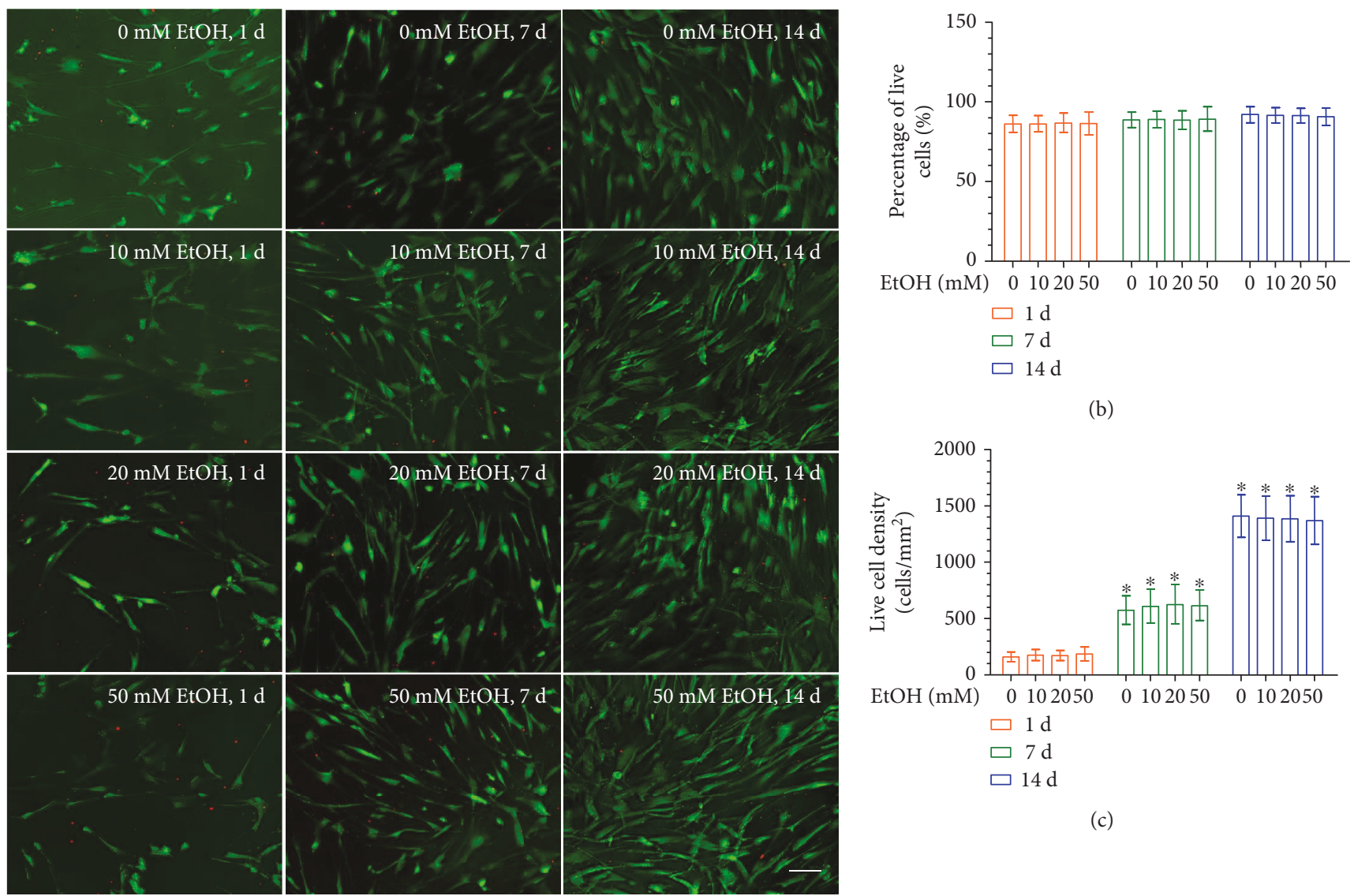

(b)

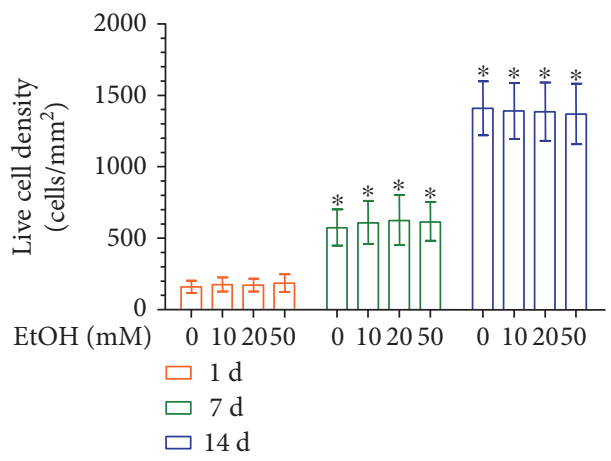

(c)

(a)

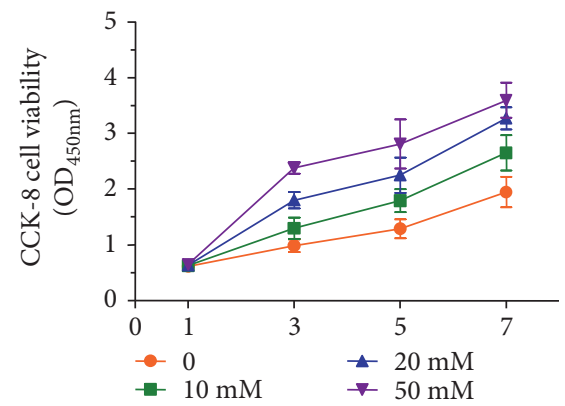

(d)

FIGURE 2: Effect of EtOH on cell viability and proliferation of DPCs. (a) Representative live/dead images of EtOH-treated DPCs after days 1, 7 , and 14 of culture. Live cells were stained in green and dead cells were stained in red. In all four groups, live cells were abundant, and dead cells were few (scale bar $=50 \mu \mathrm{m}$ ). (b) Percentage of live cells of DPCs was around 90\%. Data represent mean \pm SD of 3 experiments with triplicates. (c) EtOH increased the cell proliferation. Data represent mean \pm SD of 3 experiments with triplicates. ${ }^{*} p<0.05$ versus control group.

day 14 of odontogenic culture. The absorbance of the solutions was read at $570 \mathrm{~nm}$ using a UV-visible light spectrophotometer.

2.8. Statistical Analysis. All experiments were repeated at least three times. Data are expressed as mean \pm standard deviation (SD). Results of at least three independent experiments (always performed with cells isolated from different donors) were compared by one-way ANOVA. Differences between groups were evaluated with Tukey's posttest. $p$ values $<0.05$ were considered significant.

\section{Results}

3.1. Identification of Stem Cell Phenotypic Markers in Primary DPCs. The surface markers of DPCs were analyzed using flow cytometry. Consistent with other mesenchymal stem cell populations (Figure 1), the majority of DPCs exhibited intense expression of mesenchymal surface molecular markers (CD73-99.2\%, CD90-99.6\%, and CD105-99.3\%). On the other hand, DPCs exhibited weak expression of surface markers (CD34-0.2\%), indicating that the DPCs contained mesenchymal progenitors. 

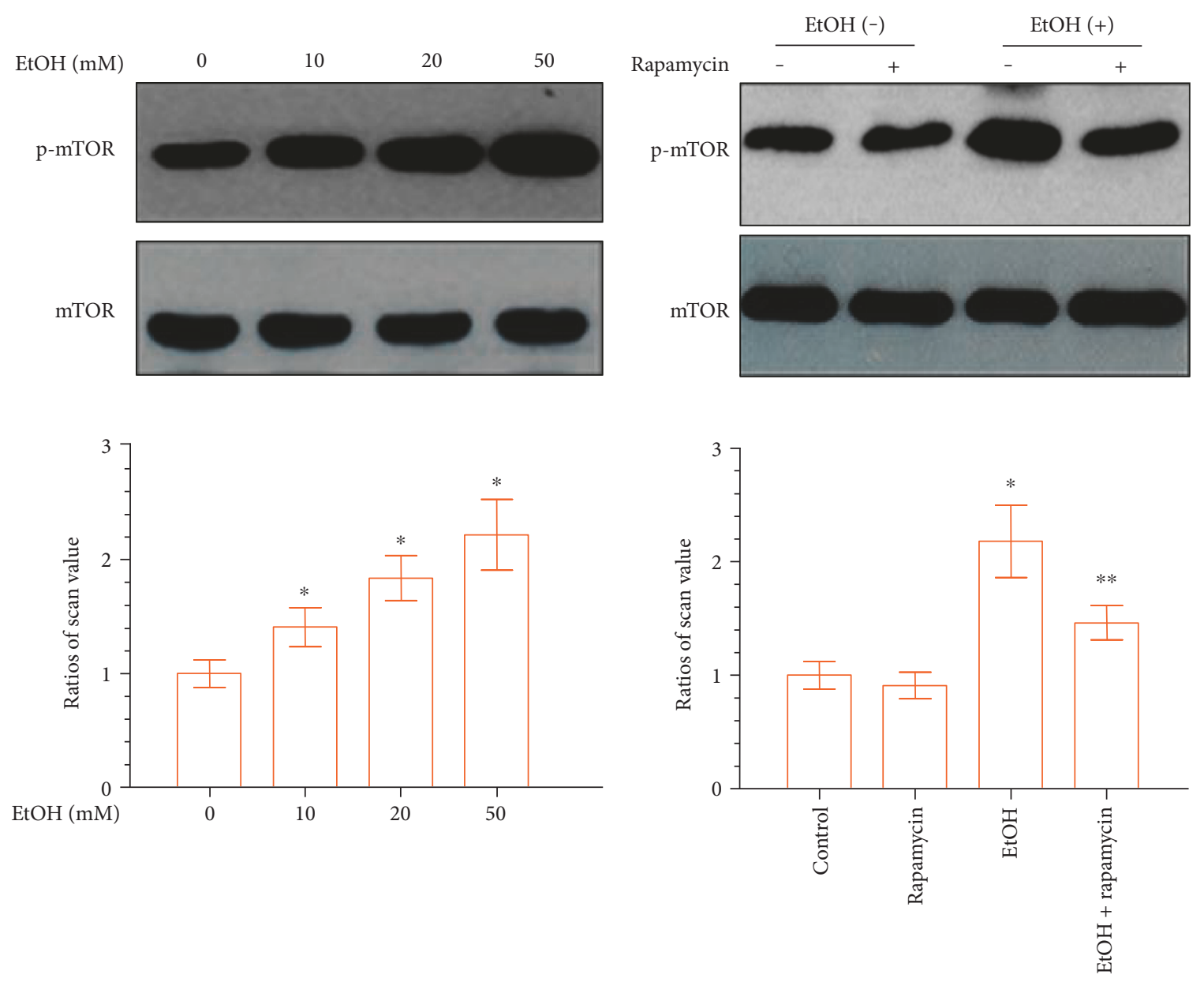

(a)

(b)

FIGURE 3: EtOH upregulates mTOR phosphorylation in DPCs in a dose-dependent manner. (a) DPCs were treated with different concentrations of EtOH for 24 hours. Lower panels show the ratios of band densities of phosphor-mTOR to mTOR. (b) Confluent DPCs cells were preincubated with the mTOR inhibitor rapamycin $(50 \mathrm{mM})$ for 1 hour before treatment with EtOH. Rapamycin decreases EtOH-induced mTOR phosphorylation. Data represent mean \pm SD of 3 experiments with triplicates. ${ }^{*} p<0.05$ versus control group. ${ }^{* *} p<0.05$ versus EtOH group.

3.2. DPC Viability and Cell Proliferation. In order to evaluate the effects of EtOH treatment on DPC cytocompatibility, cellular viability was assessed using live/dead staining after 24 hours of culture. Representative live/dead staining images are shown in Figure 2(a). There were numerous live cells (stained green) and a few dead cells (stained red). In Figure 2(b), the percentages of live cells in all four groups were approximately $90 \%$ and were not significantly different among the three doses of EtOH $(p>0.1)$. Cell number significantly increased from day 1 to day 14, most likely due to cell proliferation (Figure 2(c)). The concentrations of EtOH of up to $50 \mathrm{mM}$ enhanced cell proliferation compared to the untreated DPCs (Figure 2(d)). Overall, these results demonstrate that $50 \mathrm{mM} \mathrm{EtOH}$, used in the other experiments of the present study, was not cytotoxic to DPCs.

3.3. mTOR Activation in Response to EtOH in DPCs. To determine whether the mTOR signaling pathway is involved in the EtOH-mediated differentiation of DPCs, DPCs were treated with EtOH for 24 hours. EtOH treatment elevated the expression levels of phospho-mTOR signaling with increasing doses, compared to the control group, as shown in Figure 3(a). The small molecule rapamycin has been widely used as a selective mTOR inhibitor [28]. We used rapamycin to prevent $\mathrm{mTOR}$ phosphorylation in response to EtOH. As depicted in Figure 3(b), EtOH-induced phospho-mTOR was downregulated following pretreatment with $50 \mathrm{mM}$ rapamycin. These results confirmed that in DPCs EtOH triggers the activation of the mTOR pathway as confirmed by the inhibitory action of rapamycin.

3.4. EtOH-Induced mTOR Activation Increases Alkaline Phosphatase Activity, Alkaline Phosphatase mRNA Expression, and Mineralization. To understand whether EtOH affects the odontogenic differentiation of DPCs, an ALP activity assay was performed. To this end, ALP activity significantly decreased in the EtOH-treated group until day 14, compared to the control group (Figure 4(a)). The decreased ALP activity 


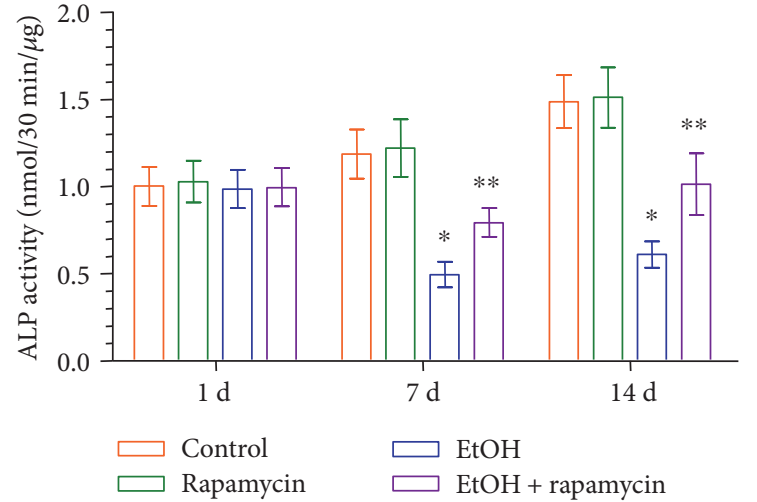

(a)
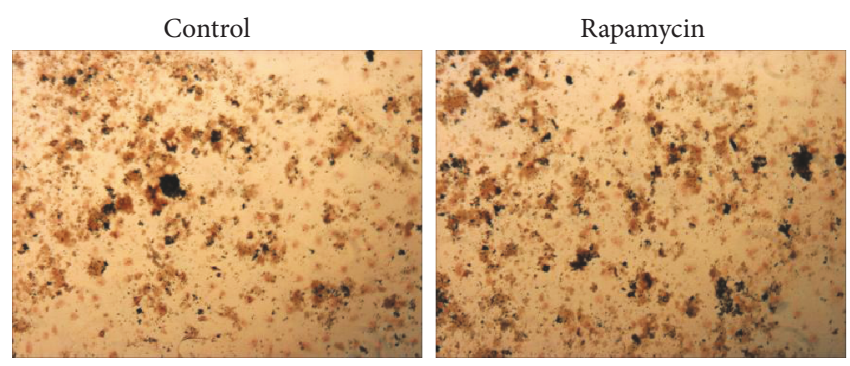

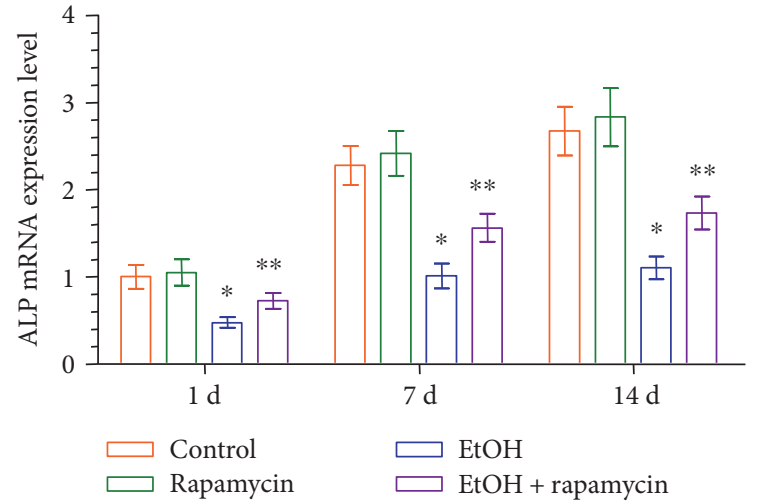

(b)
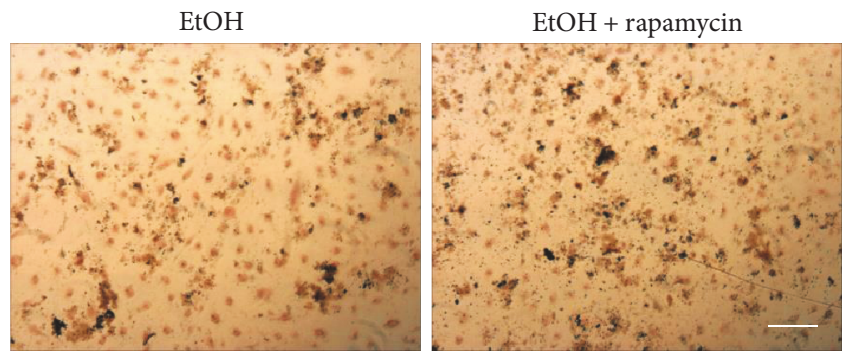

(c)

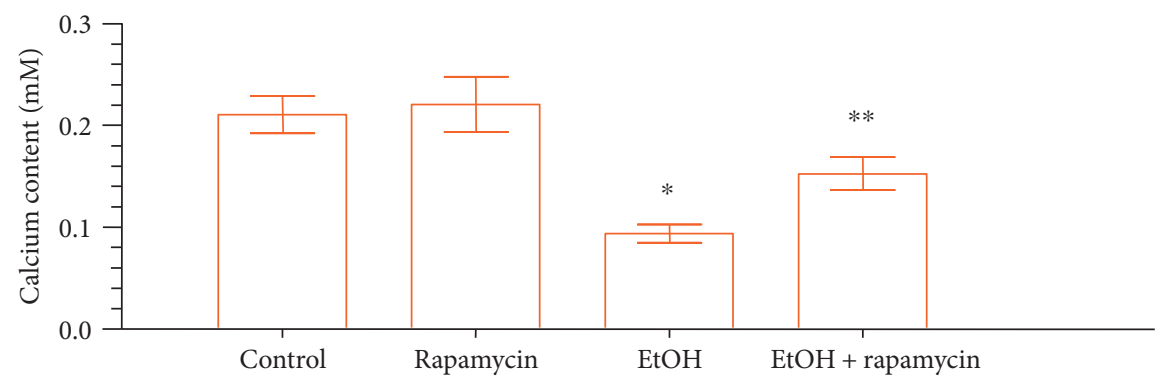

(d)

FIGURE 4: Effect of EtOH-induced ALP activity and mineralized nodule formation in DPCs. (a, b) DPCs were treated with EtOH (50 mM) in the absence or presence of rapamycin $(50 \mathrm{mM}$, pretreatment for $1 \mathrm{~h}$ ). Cells were retreated every 3 days. ALP activity (a) and ALP mRNA expression (b) were measured at each time point. (c) DPCs were cultured in osteogenic induction medium for 14 days, and the mineralized nodule formation was assessed by von Kossa staining (scale bar $=100 \mu \mathrm{m}$ ). (d) On 14 days, the calcium content was determined. Data represent mean $\pm \mathrm{SD}$ of 3 experiments with triplicates. ${ }^{*} p<0.05$ versus control group. ${ }^{* *} p<0.05$ versus EtOH group.

was significantly enhanced following pretreatment with rapamycin. The results of the ALP mRNA expression were consistent with ALP assay (Figure 4(b)). Next, we investigated the mineralized nodule formation, an index of terminal odontoblastic differentiation, in DPCs after $14 \mathrm{~d}$ of incubation with $\mathrm{EtOH}$ in the absence or presence of rapamycin. Treatment of DPCs with EtOH decreased the mineralized nodule formation and calcium content. Conversely, an increase in calcified nodule formation (Figure 4(c)) and calcium content (Figure 4(d)) was observed in cells treated with $\mathrm{EtOH}$ in the presence of rapamycin. These results suggest that the downstream effects of EtOH on the odontoblastic maturation of DPCs were mediated through the activation of the mTOR signaling pathway.

3.5. EtOH Triggers DPC Odontoblastic Differentiation in an mTOR-Dependent Manner. To further investigate the effects of EtOH on odontoblastic differentiation of DPCs, cells were exposed to EtOH for 1, 7, and $14 \mathrm{~d}$. EtOH markedly downregulated the mRNA expression of critical odontoblastic genes including DSPP, DMP-1, Runx2, and OCN mRNA. In contrast, expression of these genes was significantly elevated by the addition of rapamycin prior to $\mathrm{EtOH}$ treatment (Figure 5). These results further indicate that MTOR is a key mediating factor controlling EtOH-induced odontoblastic differentiation of DPCs.

\section{Discussion}

The long-term detrimental effects of alcoholism on bone mass have been relatively well established [29]. Previous studies showed that heavy chronic alcohol consumption is associated with a variety of risk factors that may contribute to the pathogenesis of bone disease, including malabsorption, hypogonadism, poor nutrition, vitamin $\mathrm{D}$ deficiency, liver 

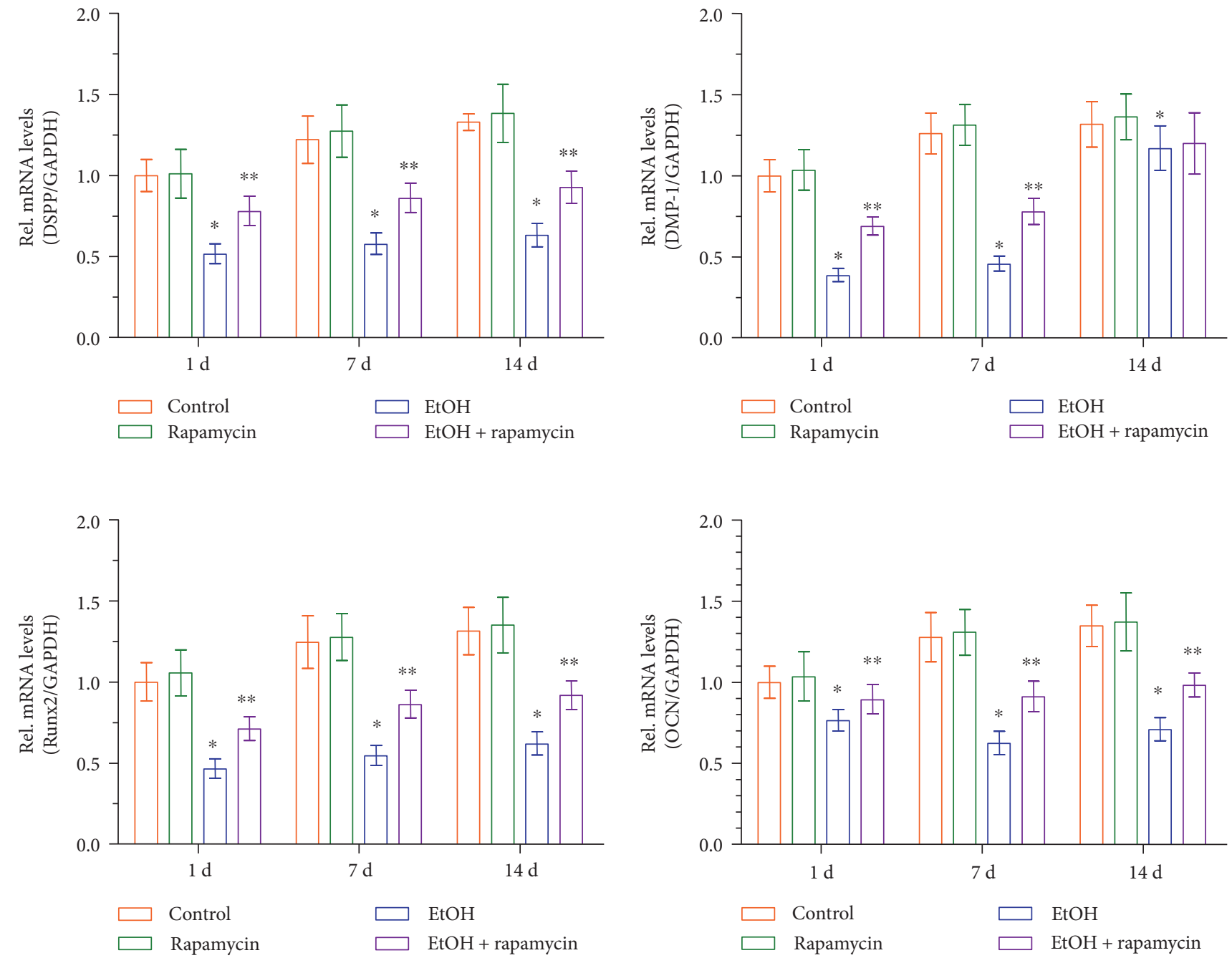

FIGURE 5: Effects of EtOH treatment on the odontoblastic differentiation of DPCs. DPCs cells were pre-incubated with rapamycin (50 mM) for 1 hour before treatment with EtOH. The mRNA expression of DSPP, DMP-1, Runx2, and OCN was analyzed using real-time RT-PCR. Data represent mean \pm SD of 3 experiments with triplicates. ${ }^{*} p<0.05$ versus control group. ${ }^{* *} p<0.05$ versus EtOH group.

disease, and parathyroid dysfunction [30]. The effect of alcohol on cell proliferation has been reported for various types of cells [31-33]. Our observations in the present study indicate that alcohol treatment was able to increase the proliferation rate of DPCs. Furthermore, The concentrations of $\mathrm{EtOH}$ of up to $50 \mathrm{mM}$ was found to not alter the viability of DPCs. Liu et al. reported that treatment of marrow mesenchymal stem cells with alcohol led to an increase in cell proliferation as determined by the methylthiazolyldiphenyl-tetrazolium bromide assay [34]. The effect of EtOH on the proliferation of DPCs is also consistent with that reported for mouse bone marrow mesenchymal stem cells.

Alcohol has been reported to activate mTOR in bone marrow mesenchymal cells [34]. Interestingly, this study showed that the addition of rapamycin, a widely used mTOR inhibitor [35], attenuated the alcohol-induced responses. These results suggested that alcohol decreased the differentiation and mineralization of osteoblast-like cells via the activation of mTOR signaling. To investigate whether
mTOR signaling pathway is involved in EtOH-induced odontogenic differentiation of DPCs, we assessed the activation of mTOR signaling in EtOH-treated DPCs under conditions designed to induce odontoblastic differentiation and its role in odontoblastic differentiation with the use of mTOR inhibitors. Among the concentration gradients in our experiment, we found that $50 \mathrm{mM} \mathrm{EtOH}$ significantly upregulated phospho-mTOR activity in DPCs. Thus, this concentration was selected for subsequent experiments in the present study. Rapamycin was also used to test the effect of alcohol on mTOR phosphorylation. The phosphorylation of mTOR was partially inhibited by rapamycin. The mechanism of how EtOH regulates the odontoblast differentiation of DPCs is complicated and has not yet been elucidated completely. In addition, whether mTOR signaling participates in odontoblast differentiation of DPCs has not yet been elucidated completely.

DPCs from dental pulp tissues represent a population of mesenchymal stem/progenitor cells. The high proliferative potential of DPCs makes this population of cells suitable 
for cell-based regeneration and especially for dentin repair. DPCs could form the dentin-like tissues and bone-like tissues in vivo [36]. Several studies have showed that dentin-like tissue formation is associated with DPCs [37-39]. ALP activity is most often used as an early marker of odontoblastic differentiation and plays an important role in dentin-like tissue formation. In the present study, greater ALP activity and ALP mRNA were achieved when the mTOR signaling pathway was inhibited by rapamycin. Consistent with this finding, more mineralized nodules were also observed in DPCs treated with rapamycin at the late stage of odontoblastic differentiation. These results indicate that the mTOR signaling pathway is partly controlling the effects of EtOH on the odontoblastic differentiation of DPCs.

Furthermore, the gene expression levels of the related odontoblastic markers such as DSPP, DMP-1, Runx2, and OCN were measured to investigate the effects of mTOR signaling on the differentiation ability of odontoblasts in vitro. Runx2, of the runt domain gene family, is an essential transcription factor that controls bone and tooth development by regulating osteoblast and odontoblast differentiation $[40,41]$. DSPP and DMP-1 are members of the small integrin-binding ligand N-linked glycoprotein (SIBLING) family. DSPP was originally considered to be dentinspecific. Although several studies have recently shown its expression in bone [42-44], DSPP remains a major marker of odontoblastic differentiation. In addition, DMP-1 is essential for the mineralization of bone and dentin [45, 46]. Furthermore, OCN can be expressed by odontoblasts and is present in the dentin matrix, and it is also thought to be a reparative molecule within the dental pulp [47]. In the present study, DSPP, DMP-1, Runx2, and OCN mRNA levels were downregulated in the EtOH-treated DPCs. However, rapamycin significantly reversed the EtOHinduced downregulation of DSPP, DMP-1, Runx2, and OCN mRNAs. This provided further evidence that the mTOR signaling pathway plays an important role in EtOHmediated DPC odontoblastic differentiation. Our study provides a basis that high doses of alcohol may be detrimental to DPCs' repair and regenerative capability.

However, the level of odontogenic differentiation of DPCs can be regulated also by other important factors. Paduano et al. demonstrated that hydrogel scaffolds derived from bone extracellular matrix (bECM) promoted odontogenic differentiation of dental pulp stem cells in the absence of external inducers, and these scaffolds could be combined with osteo/odontogenic medium or growth factors [48]. Qu and Liu demonstrated that gelatin/bioactive glass hybrid scaffolds provided an excellent environment for dental pulp stem cells for odontogenic differentiation [49]. Moreover, dental-derived mesenchymal stem cells exhibited a predisposition toward other phenotypes when cultured in the appropriate media [50-53]. Interestingly, the poliphenolic fractions isolated from the beer brewing process enhanced the antioxidant and antitumor activity. Further study is needed to determine whether the positive bioactive compounds of beer and other agents could regulate the odontogenic differentiation of DPCs and their underlying mechanisms.
In conclusion, the present study demonstrated for the first time that $\mathrm{EtOH}$ can downregulate the odontoblastic differentiation of DPCs through activation of the mTOR signaling pathway, suggesting that EtOH plays an important role during the odontoblastic differentiation. Regenerating dentin is an important target for the treatment of dental pulp exposure [54]. The primary objective of vital pulp therapy is to maintain the health of pulp tissues and to stimulate the remaining pulp to regenerate the dentin-pulp complex [55]. Therefore, the aim of the present study was to investigate the potential effect of heavy EtOH consumption on pulp therapy as a factor that needs to be considered in clinical practice. Further research is needed to fully understand and support the clinical indications suggesting that heavy alcoholic consumption should be discouraged during vital pulp therapy in adults.

\section{Conflicts of Interest}

The authors declare that they have no competing interests.

\section{Authors' Contributions}

Wei Qin and Qi-Ting Huang contributed equally as co-first authors to this study.

\section{Acknowledgments}

This study was in part supported by NIH R01 DE14190 (Hockin H. K. Xu), the University of Maryland School of Dentistry bridge fund (Hockin $\mathrm{H}$. K. Xu), the Guandong Natural Science Foundation (2015A030310079) (Wei Qin), and the Young Teacher Training Program of Sun Yat-sen University (15ykpy40) (Wei Qin).

\section{References}

[1] P. E. Molina, J. D. Gardner, F. M. Souza-Smith, and A. M. Whitaker, "Alcohol abuse: critical pathophysiological processes and contribution to disease burden," Physiology (Bethesda), vol. 29, no. 3, pp. 203-215, 2014.

[2] J. M. Barragry, R. G. Long, M. W. France, M. R. Wills, B. J. Boucher, and S. Sherlock, "Intestinal absorption of cholecalciferol in alcoholic liver disease and primary biliary cirrhosis," Gut, vol. 20, no. 7, pp. 559-564, 1979.

[3] D. D. Bikle, H. K. Genant, C. Cann, R. R. Recker, B. P. Halloran, and G. J. Strewler, "Bone disease in alcohol abuse," Annals of Internal Medicine, vol. 103, no. 1, pp. 42-48, 1985.

[4] R. T. Turner, "Skeletal response to alcohol," Alcoholism, Clinical and Experimental Research, vol. 24, no. 11,pp. 1693-1701, 2000.

[5] J. D. Pedrera-Zamorano, J. M. Lavado-Garcia, R. RonceroMartin, J. F. Calderon-Garcia, T. Rodriguez-Dominguez, and M. L. Canal-Macias, "Effect of beer drinking on ultrasound bone mass in women," Nutrition, vol. 25, no. 10, pp. 10571063, 2009.

[6] K. L. Tucker, R. Jugdaohsingh, J. J. Powell et al., "Effects of beer, wine, and liquor intakes on bone mineral density in older men and women," The American Journal of Clinical Nutrition, vol. 89, no. 4, pp. 1188-1196, 2009. 
[7] R. Jugdaohsingh, M. A. O'Connell, S. Sripanyakorn, and J. J. Powell, "Moderate alcohol consumption and increased bone mineral density: potential ethanol and non-ethanol mechanisms," The Proceedings of the Nutrition Society, vol. 65, no. 3, pp. 291-310, 2006.

[8] J. Alvisa-Negrin, E. Gonzalez-Reimers, F. Santolaria-Fernandez et al., "Osteopenia in alcoholics: effect of alcohol abstinence," Alcohol and Alcoholism, vol. 44, no. 5, pp. 468-475, 2009.

[9] L. J. Dominguez, R. Scalisi, and M. Barbagallo, "Therapeutic options in osteoporosis," Acta Biomed, vol. 81, no. 1, pp. 5565, 2010.

[10] P. Hadji, R. Coleman, and M. Gnant, "Bone effects of mammalian target of rapamycin (mTOR) inhibition with everolimus," Critical Reviews in Oncology/Hematology, vol. 87, no. 2, pp. 101-111, 2013.

[11] C. Chen, K. Akiyama, D. Wang et al., "mTOR inhibition rescues osteopenia in mice with systemic sclerosis," The Journal of Experimental Medicine, vol. 212, no. 1, pp. 73-91, 2015.

[12] J. J. Chidiac, B. Al-Asmar, K. Rifai, S. J. Jabbur, and N. E. Saadé, "Inflammatory mediators released following application of irritants on the rat injured incisors. The effect of treatment with anti-inflammatory drugs," Cytokine, vol. 46, no. 2, pp. 194-200, 2009.

[13] A. J. Sloan and A. J. Smith, "Stem cells and the dental pulp: potential roles in dentine regeneration and repair," Oral Diseases, vol. 13, no. 2, pp. 151-157, 2007.

[14] A. J. Smith, "Vitality of the dentin-pulp complex in health and disease: growth factors as key mediators," Journal of Dental Education, vol. 67, no. 6, pp. 678-689, 2003.

[15] Y. C. Hwang, I. N. Hwang, W. M. Oh, J. C. Park, D. S. Lee, and H. H. Son, "Influence of TGF-betal on the expression of BSP, DSP, TGF-beta1 receptor I and Smad proteins during reparative dentinogenesis," Journal of Molecular Histology, vol. 39, no. 2, pp. 153-160, 2008.

[16] P. Papagerakis, A. Berdal, M. Mesbah et al., "Investigation of osteocalcin, osteonectin, and dentin sialophosphoprotein in developing human teeth," Bone, vol. 30 , no. 2, pp. 377385, 2002.

[17] J. Yang, L. Ye, T. Q. Hui et al., "Bone morphogenetic protein 2 -induced human dental pulp cell differentiation involves p38 mitogen-activated protein kinase-activated canonical WNT pathway," International Journal of Oral Science, vol. 7, no. 2, pp. 95-102, 2015.

[18] F. He, Z. Yang, Y. Tan et al., "Effects of Notch ligand Delta1 on the proliferation and differentiation of human dental pulp stem cells in vitro," Archives of Oral Biology, vol. 54, no. 3, pp. 216-222, 2009.

[19] W. Qin, F. Yang, R. Deng et al., "Smad 1/5 is involved in bone morphogenetic protein-2-induced odontoblastic differentiation in human dental pulp cells," Journal of Endodontia, vol. 38, no. 1, pp. 66-71, 2012.

[20] Y. Y. Chen, S. T. He, F. H. Yan et al., "Dental pulp stem cells express tendon markers under mechanical loading and are a potential cell source for tissue engineering of tendon-like tissue," International Journal of Oral Science, vol. 8, no. 4, pp. 213-222, 2016.

[21] L. Wang, C. Zhang, C. Li et al., "Injectable calcium phosphate with hydrogel fibers encapsulating induced pluripotent, dental pulp and bone marrow stem cells for bone repair," Materials Science \& Engineering. C, Materials for Biological Applications, vol. 69, pp. 1125-1136, 2016.
[22] P. Wang, X. Liu, L. Zhao et al., "Bone tissue engineering via human induced pluripotent, umbilical cord and bone marrow mesenchymal stem cells in rat cranium," Acta Biomaterialia, vol. 18, pp. 236-248, 2015.

[23] J. Guo, W. Qin, Q. Xing et al., "TRIM33 is essential for osteoblast proliferation and differentiation via BMP pathway," Journal of Cellular Physiology, vol. 232, pp. 3158-3169, 2017.

[24] C. G. Pfeifer, A. Karl, A. Berner et al., "Expression of BMP and actin membrane bound inhibitor is increased during terminal differentiation of MSCs," Stem Cells International, vol. 2016, Article ID 2685147, 9 pages, 2016.

[25] T. Nakamura, Y. Chiba, M. Naruse, K. Saito, H. Harada, and S. Fukumoto, "Globoside accelerates the differentiation of dental epithelial cells into ameloblasts," International Journal of Oral Science, vol. 8, no. 4, pp. 205-212, 2016.

[26] W. Qin, P. Liu, R. Zhang et al., "JNK MAPK is involved in BMP-2-induced odontoblastic differentiation of human dental pulp cells," Connective Tissue Research, vol. 55, no. 3, pp. 217-224, 2014.

[27] W. Qin, Z. M. Lin, R. Deng et al., "p38a MAPK is involved in BMP-2-induced odontoblastic differentiation of human dental pulp cells," International Endodontic Journal, vol. 45, no. 3, pp. 224-233, 2012.

[28] A. R. Martin, R. A. Pollack, A. Capoferri, R. F. Ambinder, C. M. Durand, and R. F. Siliciano, "Rapamycin-mediated mTOR inhibition uncouples HIV-1 latency reversal from cytokineassociated toxicity," The Journal of Clinical Investigation, vol. 127, no. 2, pp. 651-656, 2017.

[29] D. Feskanich, S. A. Korrick, S. L. Greenspan, H. N. Rosen, and G. A. Colditz, "Moderate alcohol consumption and bone density among postmenopausal women," Journal of Women's Health, vol. 8, no. 1, pp. 65-73, 1999.

[30] M. J. Kim, M. S. Shim, M. K. Kim et al., "Effect of chronic alcohol ingestion on bone mineral density in males without liver cirrhosis," The Korean Journal of Internal Medicine, vol. 18, no. 3, pp. 174-180, 2003.

[31] J. Lee, M. S. Shin, M. O. Kim et al., “Apple ethanol extract promotes proliferation of human adult stem cells, which involves the regenerative potential of stem cells," Nutrition Research, vol. 36, no. 9, pp. 925-936, 2016.

[32] P. D. Narayanan, S. K. Nandabalan, and L. S. Baddireddi, "Role of STAT3 phosphorylation in ethanol-mediated proliferation of breast cancer cells," Journal of Breast Cancer, vol. 19, no. 2, pp. 122-132, 2016.

[33] H. S. Kim, S. J. Kim, J. Bae et al., “The p90rsk-mediated signaling of ethanol-induced cell proliferation in HepG2 cell line," The Korean Journal of Physiology and Pharmacology, vol. 20, no. 6, pp. 595-603, 2016.

[34] Y. Liu, X. Kou, C. Chen et al., "Chronic high dose alcohol induces osteopenia via activation of mTOR signaling in bone marrow mesenchymal stem cells," Stem Cells, vol. 34, no. 8, pp. 2157-2168, 2016.

[35] K. J. Kinghorn, S. Gronke, J. I. Castillo-Quan et al., “A drosophila model of neuronopathic gaucher disease demonstrates lysosomal-autophagic defects and altered mTOR signalling and is functionally rescued by rapamycin," Journal of Neuroscience, vol. 36, no. 46, pp. 11654-11670, 2016.

[36] G. T. Huang, S. Gronthos, and S. Shi, "Mesenchymal stem cells derived from dental tissues vs. those from other sources: their biology and role in regenerative medicine," Journal of Dental Research, vol. 88, no. 9, pp. 792-806, 2009. 
[37] J. Caton, N. Bostanci, E. Remboutsika, C. De Bari, and T. A. Mitsiadis, "Future dentistry: cell therapy meets tooth and periodontal repair and regeneration," Journal of Cellular and Molecular Medicine, vol. 15, no. 5, pp. 1054-1065, 2011.

[38] A. A. Volponi, Y. Pang, and P. T. Sharpe, "Stem cell-based biological tooth repair and regeneration," Trends in Cell Biology, vol. 20, no. 12, pp. 715-722, 2010.

[39] N. E. Abou, W. Chrzanowski, V. M. Salih, H. W. Kim, and J. C. Knowles, "Tissue engineering in dentistry," Journal of Dentistry, vol. 42, no. 8, pp. 915-928, 2014.

[40] B. Jin and P. H. Choung, "Recombinant human plasminogen activator inhibitor-1 accelerates odontoblastic differentiation of human stem cells from apical papilla," Tissue Engineering Part A, vol. 22, no. 9-10, pp. 721-732, 2016.

[41] F. Posa, A. Benedetto, and G. Colaianni, "Vitamin D effects on osteoblastic differentiation of mesenchymal stem cells from dental tissues," Stem Cells International, vol. 2016, Article ID 9150819, 9 pages, 2016.

[42] O. Baba, C. Qin, J. C. Brunn et al., "Detection of dentin sialoprotein in rat periodontium," European Journal of Oral Sciences, vol. 112, no. 2, pp. 163-170, 2004.

[43] J. K. Kim, R. Shukla, L. Casagrande et al., "Differentiating dental pulp cells via RGD-dendrimer conjugates," Journal of Dental Research, vol. 89, no. 12, pp. 1433-1438, 2010.

[44] I. S. Kim, Y. M. Song, and S. J. Hwang, "Osteogenic responses of human mesenchymal stromal cells to static stretch," Journal of Dental Research, vol. 89, no. 10, pp. 1129-1134, 2010.

[45] L. W. Fisher, "DMP1 and DSPP: evidence for duplication and convergent evolution of two SIBLING proteins," Cells, Tissues, Organs, vol. 194, no. 2-4, pp. 113-118, 2011.

[46] A. Balic and M. Mina, "Identification of secretory odontoblasts using DMP1-GFP transgenic mice," Bone, vol. 48, no. 4, pp. 927-937, 2011.

[47] N. Goto, K. Fujimoto, and S. Fujii, "Role of MSX1 in osteogenic differentiation of human dental pulp stem cells," Stem Cells International, vol. 2016, Article ID 8035759, 13 pages, 2016.

[48] F. Paduano, M. Marrelli, L. J. White, K. M. Shakesheff, and M. Tatullo, "Odontogenic differentiation of human dental pulp stem cells on hydrogel scaffolds derived from decellularized bone extracellular matrix and collagen type I," PLoS One, vol. 11, no. 2, article e148225, 2016.

[49] T. Qu and X. Liu, "Nano-structured gelatin/bioactive glass hybrid scaffolds for the enhancement of odontogenic differentiation of human dental pulp stem cells," Journal of Materials Chemistry. B, Materials for Biology and Medicine, vol. 1, no. 37, pp. 4764-4772, 2013.

[50] M. Marrelli, F. Paduano, and M. Tatullo, "Human periapical cyst-mesenchymal stem cells differentiate into neuronal cells," Journal of Dental Research, vol. 94, no. 6, pp. 843852, 2015.

[51] M. Tatullo, M. Marrelli, K. M. Shakesheff, and L. J. White, "Dental pulp stem cells: function, isolation and applications in regenerative medicine," Journal of Tissue Engineering and Regenerative Medicine, vol. 9, no. 11, pp. 1205-1216, 2015.

[52] C. Gandia, A. Arminan, J. M. Garcia-Verdugo et al., "Human dental pulp stem cells improve left ventricular function, induce angiogenesis, and reduce infarct size in rats with acute myocardial infarction," Stem Cells, vol. 26, no. 3, pp. 638-645, 2008.
[53] G. Carnevale, M. Riccio, A. Pisciotta et al., "In vitro differentiation into insulin-producing beta-cells of stem cells isolated from human amniotic fluid and dental pulp," Digestive and Liver Disease, vol. 45, no. 8, pp. 669-676, 2013.

[54] A. Nowicka, M. Lipski, M. Parafiniuk et al., "Response of human dental pulp capped with biodentine and mineral trioxide aggregate," Journal of Endodontia, vol. 39, no. 6, pp. 743-747, 2013.

[55] F. Schwendicke, F. Brouwer, and M. Stolpe, "Calcium hydroxide versus mineral trioxide aggregate for direct pulp capping: a cost-effectiveness analysis," Journal of Endodontia, vol. 41, no. 12, pp. 1969-1974, 2015. 

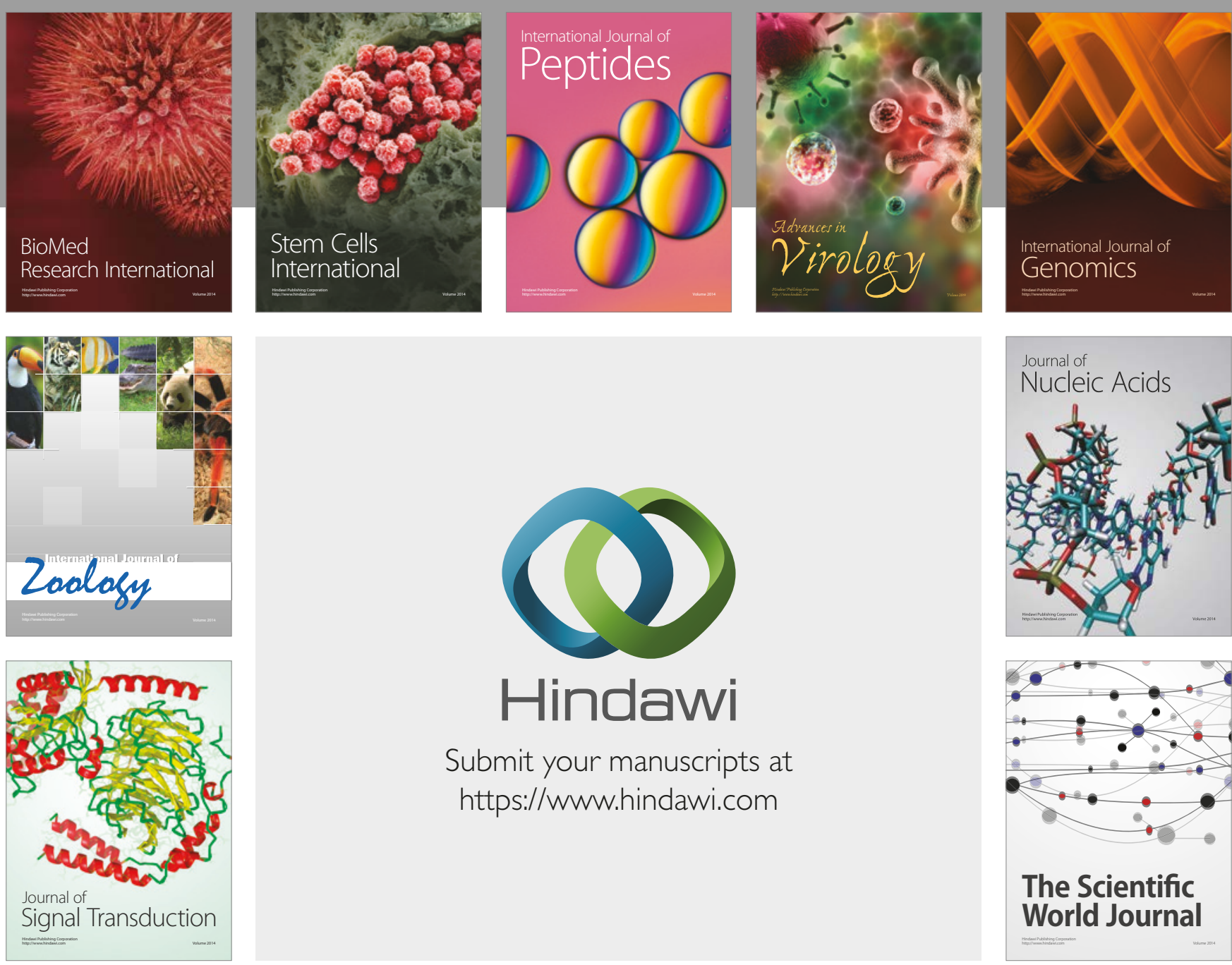

Submit your manuscripts at

https://www.hindawi.com
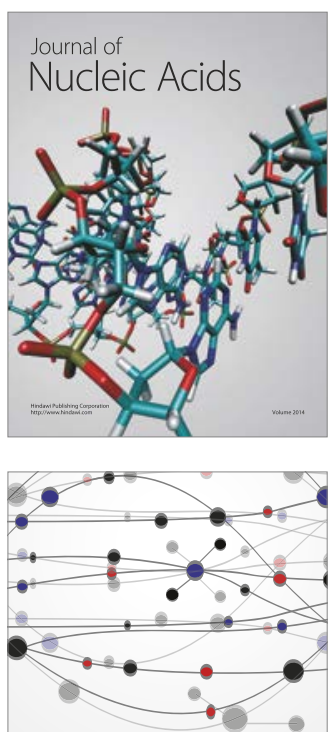

The Scientific World Journal

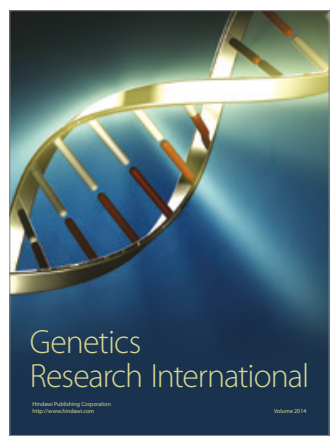

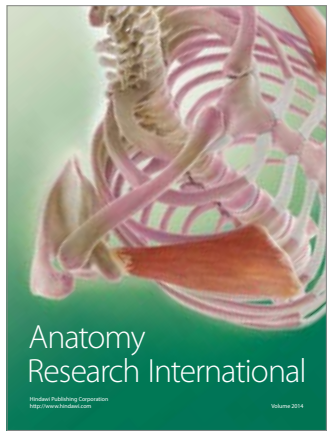

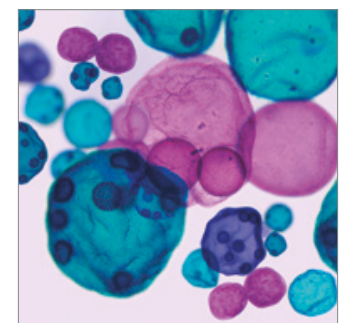

International Journal of Microbiology
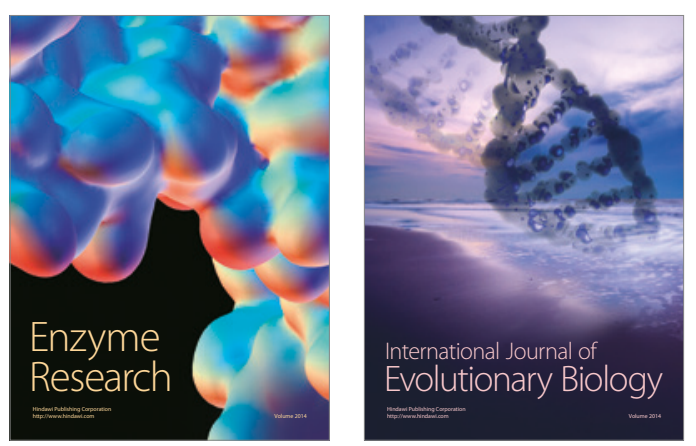
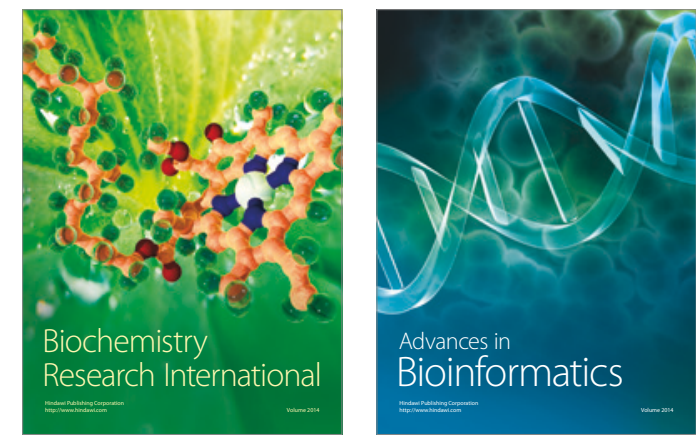

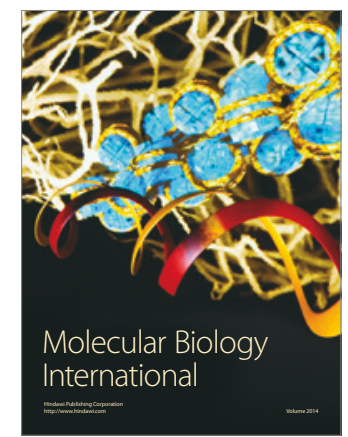

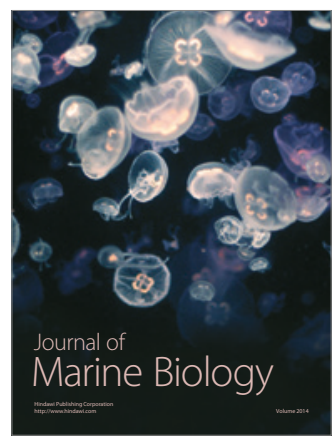

